\title{
HERAFitter project and its related studies
}

\author{
Vladimir KOLESNIKOV* \\ on behalf of the HERAFit ter team \\ Joint Institute for Nuclear Research \\ E-mail: kolesnikov.volodya@gmail.com
}

HERAFitter is an open-source package that provides a framework for the determination of the parton distribution functions (PDFs) of the proton and for many different kinds of analyses in Quantum Chromodynamics (QCD). It encodes results from a wide range of experimental measurements at hadron colliders along with corresponding theoretical predictions. The framework covers a large number of the existing methods and schemes used for PDF determination. It can be used to study the impact of new precise measurements from hadron colliders. A short overview of the package is presented in this paper together with the recent analyses published by the HERAF itter developers team.

XXVII International Symposium on Lepton Photon Interactions at High Energies

17-22 August 2015

Ljubljana, Slovenia

${ }^{*}$ Speaker. 


\section{Introduction}

According to the factorisation theorem, the cross-section of hadron collisions is a convolution of hadron PDFs with perturbatively calculable partonic reactions. PDFs and their uncertainties are crucial for precision tests of Standard Model and for searching of physics beyond Standard Model. HERAFitter is an open source QCD fit framework designed for the extraction of PDFs of the proton and different analyses in QCD. HERAFitter implements various methodologies of PDF fits and tools to asses the impact of new data on PDFs. Further information about the package,

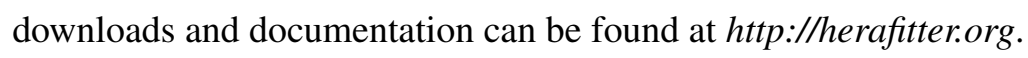

\section{Functionality}

In general PDFs are determined by a fit to experimental data for various processes. A common PDF fit workflow in HERAFitter is shown below:

- First, the PDFs are parametrised at the starting scale $Q_{0}^{2}$ below the charm mass threshold.

- Parametrised PDFs are evolved to the scale of experimental data using DGLAP formalism [1] in $\overline{M S}$ scheme. For PDF evolution several external libraries are used: QCDNUM [2] and APFEL [3].

- Evolved PDFs are convoluted with partonic crossections to get theoretical prediction of observables.

- The experimental and predicted crossections together with their corresponding errors are used to build a $\chi^{2}$ and determine the PDFs parameters.

- The $\chi^{2}$ is minimised with the MINUIT [4] program.

- The resulting PDFs are saved in formats corresponding to LHAPDF [5] or TMDlib [6].

In HERAFitter different data from $e p, p p$ and $p \bar{p}$ colliders can be used in the QCD fits to constrain PDFs in various kinematic domains: inclusive cross sections from HERA DIS and fixed target experiments, Drell-Yan, jet production data, top quark pair production. Various schemes for the treatment of heavy quark production in DIS are covered by the package: Thorne-Roberts (TR) at LO, NLO, NNLO as provided by the MSTW group, the ACOT scheme at LO and NLO as provided by CTEQ group, zero-mass (ZM) and fixed flavour number (FFN) schemes implemented in QCDNUM, FONLL scheme via APFEL. FFN scheme is also available via OPENQCDRAD [7]. Several methods for calculation of processes crossection are available in HERAFitter. The NLO and NNLO predictions could be obtained using $k$-factor or fast-grid (via APPLGRID [8] or FastNLO [9]) techniques.

The PDFs can be parametrised using several predefined functional forms:

- the standard polynomial form is the most commonly used,

- bi-log-normal distributions can be regarded as a generalization of standard polynomial form,

- Chebyshev polynomials can be effectively used for the gluon and sea distributions.

HERAFitter can work with various forms of $\chi^{2}$, e.g. using a covariance matrix or providing nuisance parameters to encode the dependence of each correlated systematic uncertainty for each measured data point: 
- covariance matrix representation

$$
\chi^{2}(m)=\sum_{i, k}\left(m_{i}-\mu_{i}\right) C_{i k}^{-1}\left(m_{k}-\mu_{k}\right)
$$

for a data point $\mu_{i}$ with a corresponding theory prediction $m_{i}$, where the covariance matrix $C_{i k}$ is given by a sum of statistical, uncorrelated and correlated systematic contributions;

- nuisance parameter representation

$$
\chi^{2}(m, b)=\sum_{i} \frac{\left[\mu_{i}-m_{i}\left(1-\sum_{j} \gamma_{j}^{i} b_{j}\right)\right]^{2}}{\delta_{i, \text { unc }}^{2} m_{i}^{2}+\delta_{i, \text { stat }}^{2} \mu_{i} m_{i}\left(1-\sum_{j} \gamma_{j}^{i} b_{j}\right)}+\sum_{j} b_{j}^{2}
$$

where, $\delta_{i, \text { stat }}$ and $\delta_{i, \text { unc }}$ are relative statistical and uncorrelated systematic uncertainties of the measurement $i, \gamma_{j}^{i}$ quantifies the sensitivity of the measurement to the correlated systematic source $j$, the function $\chi^{2}$ depends on the set of systematic nuisance parameters $b_{j}$;

- mixed form, where first two approaches are combined.

As an alternative to a complete QCD fit, several additional methods for estimation of the impact of new data on PDFs are available in HERAFitter:

Bayesian Reweighting was first proposed for PDF sets in the form of MC replicas [10] and further developed by the NNPDF Collaboration [11,12]. More recently, a method to perform Bayesian Reweighting studies using PDFs with uncertainties provided in the eigenvector representation has been also developed [13]. Both reweighting methods are implemented in HERAFitter.

Another method is profiling procedure. The profiling is performed using a $\chi^{2}$ function which includes both the experimental uncertainties and theoretical uncertainties arising from PDF variations:

$$
\chi^{2}\left(\beta_{\exp }, \beta_{t h}\right)=\sum_{i=1}^{N_{\text {data }}} \frac{\left(\sigma_{i}^{\exp }+\sum_{j} \Gamma_{i j}^{\exp } \beta_{j, \exp }-\sigma_{i}^{t h}-\sum_{k} \Gamma_{i k}^{t h} \beta_{k, t h}\right)^{2}}{\Delta_{i}^{2}}+\sum_{j} \beta_{j, \exp }^{2}+\sum_{k} \beta_{k, t h}^{2} .
$$

The correlated experimental and theoretical uncertainties are included using nuisance parameter vectors $\beta_{\text {exp }}$ and $\beta_{t h}$. Their influence on the data and theory predictions is described by the $\Gamma_{i j}^{\exp }$ and $\Gamma_{i j}^{e x p}$ matrices. The measurements and the uncorrelated experimental uncertainties are given by $\sigma_{i}^{e x p}$ and $\Delta_{i}$, respectively, and the theory predictions are $\sigma_{i}^{\text {th }}$.

\section{Recent studies using HERAFitter}

HERAFitter is actively used by experimental and theoretical high energy physics communities. There are several QCD analyses done by ATLAS and CMS collaborations using HERAF itter (for the most recent analyses see $[14,15,16]$ ).

A recent study done by HERAFitter developers team is dedicated to PDF sets at LO, NLO, NNLO accounting for correlated uncertainties between orders [17]. The sets are used to study 
cross-section ratios and their uncertainties when calculated at different orders in QCD. A reduction of the overall theoretical uncertainty is observed if correlations between the PDF sets are taken into account for the ratio of $W W$ di-boson to $Z$ boson production cross sections at the LHC.

Another new work is related to assessing of the impact of the Tevatron $W$ and $Z$ boson data on PDFs using new developments in HERAFitter [18]. Recent measurements of the $W$ boson charge asymmetry and of the $Z$ boson production cross sections, performed at the Tevatron collider in Run II by the $D 0$ and CDF collaborations, are studied using the HERAFitter framework to assess their impact on the proton PDFs. The Tevatron measurements, together with deep-inelastic scattering data from HERA, are included in a QCD analysis performed at next-to-leading order, and compared to the predictions obtained using other PDF sets from different groups. Good agreement between measurements and theoretical predictions is observed. The Tevatron data provide significant constraints on the $d$-valence quark distribution.

\section{References}

[1] G. Altarelli and G. Parisi, Asymptotic freedom in parton language, Nucl. Phys. B 126 (1977) 298.

[2] M. Botje, QCDNUM: Fast QCD Evolution and Convolution, Comput.Phys.Commun. 182 (2011) 490-532, [1005.1481].

[3] V. Bertone, S. Carrazza, and J. Rojo, APFEL: A PDF Evolution Library with QED corrections, Comput. Phys. Commun. 185 (2014) 1647-1668, [arXiv:1310.1394].

[4] F. James and M. Roos Comput. Phys. Commun. 10 (1975) 343-367.

[5] M. Whalley, D. Bourilkov, and R. Group, The Les Houches accord PDFs (LHAPDF) and LHAGLUE, hep-ph/0508110.

[6] F. Hautmann, H. Jung, M. Kramer, P. Mulders, E. Nocera, et al., TMDlib and TMDplotter: library and plotting tools for transverse-momentum-dependent parton distributions - version 1.0.0, 1408.3015.

[7] S. Alekhin, J. Blümlein, and S. Moch, "OPENQCDRAD."

[8] T. Carli et al., A posteriori inclusion of parton density functions in NLO QCD final-state calculations at hadron colliders: The APPLGRID Project, Eur. Phys. J. C66 (2010) 503-524, [0 911 . 2985].

[9] D. Britzger, K. Rabbertz, F. Stober, and M. Wobisch, New features in version 2 of the fastNLO project, 1208.3641 .

[10] W. T. Giele and S. Keller, Implications of hadron collider observables on parton distribution function uncertainties, Phys.Rev. D58 (1998) 094023, [hep-ph/ 9803393 ].

[11] R. D. Ball, V. Bertone, F. Cerutti, L. Del Debbio, S. Forte, et al., Reweighting and Unweighting of Parton Distributions and the LHC W lepton asymmetry data, Nucl.Phys. B855 (2012) 608-638, [1108.1758].

[12] NNPDF Collaboration, R. D. Ball et al., Reweighting NNPDFs: the W lepton asymmetry, Nucl.Phys. B849 (2011) 112-143, [1012.0836].

[13] G. Watt and R. Thorne, Study of Monte Carlo approach to experimental uncertainty propagation with MSTW 2008 PDFs, JHEP 1208 (2012) 052, [1205. 4024].

[14] J. Rojo et al., The PDF4LHC report on PDFs and LHC data: Results from Run I and preparation for Run II, J. Phys. G42 (2015) 103103, [arXiv: 1507.00556$].$ 
[15] CMS Collaboration, Measurement of the muon charge asymmetry in inclusive pp to WX production at $\operatorname{sqrt}(s)=8 \mathrm{TeV}$, Tech. Rep. CMS-PAS-SMP-14-022, CERN, Geneva, 2015.

[16] ATLAS Collaboration, G. Aad et al., Measurement of the forward-backward asymmetry of electron and muon pair-production in pp collisions at $\sqrt{s}=7$ TeV with the ATLAS detector, JHEP 09 (2015) 049, [arXiv:1503.03709].

[17] P. Belov et al., Parton distribution functions at LO, NLO and NNLO with correlated uncertainties between orders, Eur. Phys. J. C74 (2014), no. 10 3039, [arXiv: 1404.4234 ].

[18] S. Camarda et al., QCD analysis of W- and Z-boson production at Tevatron, Eur. Phys. J. C75 (2015), no. 9 458, [arXiv:1503.05221]. 